\title{
Negative predictive value of F-18-FDG coincidence PET in patients with Hodgkin's disease and a residual mass after therapy: a retrospective diagnostic test study
}

\author{
Dražen Huić ${ }^{1}$, Andrea Mutvar ${ }^{1}$, Sandra Kinda-Bašić ${ }^{2}$, Igor Aurer ${ }^{2}$, Martina \\ Ciglar1 ${ }^{1}$, Darko Grošev ${ }^{1}$, Ivo Radman², Boris Labar ${ }^{2}$, Damir Dodig ${ }^{1}$ \\ ${ }^{1}$ Department of Nuclear Medicine and Radiation Protection, ${ }^{2}$ Department of Internal Medicine, \\ Division of Hematology, Zagreb University Hospital Centre, Zagreb, Croatia
}

\begin{abstract}
Background. The aim of the study was to asses the negative predictive value (NPV) of FDG-PET performed with triple-head coincidence gamma camera after the first-line therapy or salvage therapy in patients with Hodgkin's disease (HD) compared by a long-term follow-up as a reference standard.

Methods. This retrospective diagnostic test study was done at the University Hospital Centre Zagreb between June 2001 and February 2008. The charts of 131 consecutive patients with Hodgkin's disease were reviewed. Seventy-three consecutive PET-negative patients (median age 28 years; range 12-80 years) with primary or recurrent biopsy confirmed lymphoma after the first-line therapy or salvage therapy were followed-up at least 12 months (median 23 months; range 12-69 months). All already performed ${ }^{18}$ F-FDG PET scans (using hybrid PET camera with triple head coincidence imaging capability within a few months after the completion of the therapy) were again visually interpreted by two board-certified nuclear medicine physicians who were blinded to any clinical or CT data. The negative predictive value of FDG-PET performed with triple-head coincidence gamma camera (Index test) was compared with a long-term follow-up as a reference standard.
\end{abstract}

Results. Out of 131 patients 73 turned-out to be PET-negative. Of those 73 PET-negative patients, 61 have been scanned after the first-line chemotherapy/radiotherapy, and only 3 of them relapsed in a follow-up (negative predictive value 0.95). Twelve patients with resistant disease have been scanned after the repeated therapy, and 4 of them relapsed in a follow-up period (negative predictive value 0.66 ).

Conclusions. This methodology with a triple-head coincidence gamma camera has a high negative predictive value. A negative PET scan can reassure patients and their doctors that the disease is not active.

Key words: Hodgkin's disease; fluorine-18-fluorodeoxyglucose; FDG; PET; therapy monitoring; prognosis; follow-up; negative predictive value

Correspondence to: Dražen Huić, MD, PhD, Department of Nuclear Medicine and Radiation Protection, Zagreb University Hospital Centre, Kišpatićeva 12, 10000 Zagreb, Croatia; Phone: +385 12388 086; Fax: +385 1 2421 874; E mail: dhuic@kbc-zagreb.hr
Conflicts of interest and source of funding: We state no conflicts of interest in this work. Our investigation was partially supported by the grant 108-1081872-2062 from the Croatian Ministry for Science, Education and Sport. 


\section{Introduction}

Hodgkin's disease (HD) is a highly curable malignancy. With the modern therapy a disease-free survival is around $80 \%$ at 5 years. ${ }^{1}$ However, HD survivors face significant medical problems related to toxicities of chemo- and radiotherapy, including secondary primary malignancies, cardiovascular and endocrine problems. ${ }^{2}$ Therefore, avoiding an unnecessary therapy is very important. $^{3}$

Accurate staging is essential for optimizing the patient's therapy but the main dilemma in assessing response at the end of the treatment is the presence of residual mass. In the large percentage of patients (> 60\%) conventional imaging methods show remaining tumour masses at the end of the therapy, although only a small percentage of these patients still have the active disease and eventually will relapse. ${ }^{1,4,5}$

Computed tomography (CT) has long been the standard procedure for staging, therapy monitoring and follow-up of lymphoma patients, but it has well-known limitations, caused by the fact that the differentiation between normal and abnormal findings is based exclusively on lymph node size, and cannot differentiate scar tissue from viable tumour in the residual mass. $3,6,7$

Metabolic imaging using fluorine-18fluorodeoxyglucose ( ${ }^{18} \mathrm{~F}$-FDG) PET provides a functional characterization of tissue and assesses tumour viability unrelated to morphologic criteria. ${ }^{8,9}$ High accuracy of FDG-PET in characterization of residual masses and early detection of recurrent disease cause this imaging method to have an important role in the management of lymphoma by enabling a more precise and accurate determination of the disease status. The accurate assessment of response at the end of the therapy is of considerable prognostic importance because it can enable physicians to withhold the unnecessary additional radiotherapy or even high-dose chemotherapy and autografting and, thus, spare the patient's acute and late toxicity.

Numerous studies and two recent metaanalyses confirm a high negative predictive value (NPV) of FDG-PET performed using dedicated PET scanners in patients with HD. $4,10-12$ The same is not true for positive FDG-PET findings and generally, in such cases a biopsy is recommended for the confirmation of presence of the active disease. $^{11-14}$

It is well known that coincidence gamma cameras have inferior sensitivity for the tumour detection in comparison to dedicated PET scanners. ${ }^{15,16}$ Therefore, some authors expressed doubts about the value of coincidence FDG-PET scanning for the response assessment in patients with lymphoma. . $^{17,18}$

The aim of this study was to asses the negative predictive value (NPV) of FDGPET performed with a triple-head coincidence gamma camera after the first-line therapy or salvage therapy in patients with HD compared by a long-term follow-up as a reference standard.

\section{Patients and methods}

\section{Design and setting}

This retrospective diagnostic test study was done at the University Hospital Centre Zagreb between June 2001 and February 2008.

\section{Patients}

One-hundred thirty-one patients with a residual mass after the treatment of HD from our or collaborating centres had a FDG-PET scan performed at our centre. All patients had pre-therapy biopsy - proven HD. Initial staging consisted of a careful 
clinical examination of peripheral lymph node areas, CT scanning of the thorax, abdomen and pelvis and a bone marrow biopsy. Those examinations that were positive prior to the treatment were repeated during restaging. Patients with a lymph node visible on a CT-scan bigger than 1.5 $\mathrm{cm}$ in the greatest diameter were considered to have a residual mass. ${ }^{19}$

Only the follow-up of 73 PET-negative patients was analyzed. In 61 patients the initial PET study was performed after the front-line therapy and in 12 after the salvage therapy. The follow-up of patients was at least 12 months (median 23 months; range 12-69 months).

\section{FDG-PET}

All PET studies were performed using IRIX hybrid PET camera (Philips Medical System, USA) with triple head coincidence imaging capability and equipped with parallel slat collimators. Its improved electronics for the coincidence detection allows the detectors to reject any events that normally would have caused pile-up and mispositioning. A detailed system description and performance characteristics are given elsewhere. ${ }^{20,21}$

About $370 \mathrm{MBq}(10 \mathrm{mCi})$ of ${ }^{18} \mathrm{~F}-\mathrm{FDG}$ was administered intravenously to each patient after overnight fasting. Patients received a diuretic to minimize artefacts due to urinary stasis and were kept well hydrated. Between injection and scanning patients lied still to avoid FDG muscular uptake. Sixty minutes post injection two tomographic acquisitions of the neck, thorax, abdomen and groins were done, 30 min duration each, using angular step $3^{0}$, with all three camera heads making full $360^{\circ}$ rotation in rectangular configuration (heads $2 \& 3$ parallel to each other, head 1 perpendicular to heads $2 \& 3$ ). After the acquisition, raw list mode data were rebinned into SPECT-like projections (matrix: 128x128, zoom=1.0) using single slice rebinning algorithm (axial acceptance angle $12^{\circ}$ ). The images were iteratively reconstructed. No attenuation correction was used.

\section{Interpretation of ${ }^{18} \mathrm{~F}-\mathrm{FDG}$ PET scans}

All already performed ${ }^{18}$ F-FDG PET scans were visually interpreted again by two board-certified nuclear medicine physicians (D.H., with 9 years of FDG-PET experience; A.M. with 5 years FGD-PET experience) who were blinded to any clinical or CT data. A positive result was defined as the focal activity higher than that of surrounding background tissue not located in areas of physiological ${ }^{18} \mathrm{~F}$-FDG uptake, without similar activity seen on the contra lateral side. A negative result was defined as no abnormal ${ }^{18} \mathrm{~F}$-FDG uptake at any side.

\section{Ethics}

A signed informed consent for imaging and using the patient's data for the further research was obtained from all patients. The study was approved by the Ethics Committee of University Hospital Centre, Zagreb.

\section{Statistical analyses}

The values are expressed as negative predictive values (NPV) with 95\% confidence intervals (CI) by using the exact binomial method.

\section{Results}

Out of total 131 patients 58 patients turnedout to be PET-positive, and were not included in the study. The demographic and clinical data of 73 PET-negative patients are presented in Table 1 . Their median age was 28 years (range, 12-80 years). At diag- 
nosis 3 patients were in stage I, 46 in stage II, 16 in stage III and 8 in stage IV. ABVD (doxorubicin, bleomycin, vinblastine, and dacarbazine) was the most frequently used front-line treatment. Patients in stage I and II generally received 4 cycles of ABVD and involved-field irradiation and those in stage III and IV 6 to 8 cycles of ABVD. Patients who had a negative PET scan received no further antitumor therapy until relapse.

Flow diagram of our patients is presented in Figure 1. Seven patients (out of 73) relapsed $(\mathrm{NPV}=0.90 ; \mathrm{CI}$ : 0.81-0.96), three (out of 61 patients) after the front-line treatment (NPV $=0.95 ; \mathrm{CI}: 0.86-0.99)$ and four patients (out of 12) after the salvage therapy $(\mathrm{NPV}=0.67$; CI: 0.35-0.90).

\section{Discussion}

The results show that FDG-PET performed in HD patients with a residual post treatment tumour mass using a triple-head coincidence gamma camera has a high NPV (0.90). This is especially true for front-line patients where it reaches 0.95 . Thus, our results in these patients are comparable to results obtained with dedicated PET systems with NPVs between 0.90 and $0.96 .4,7,10,22$ Lower NPV (0.66) in patients receiving salvage therapy can be expected because of a more aggressive disease course.

We have decided not to analyze the PET positive patients in detail because, after scanning, they were not treated in a uniform way. Thus, it would be very hard to discern the true positive predictive value of a positive PET scan. Furthermore, we are missing gold standard (biopsy) for the really active disease. Besides, because of physiological variants, false-positive FDG uptake is predominantly due to post-therapy inflammatory changes, which subsequently resolve. ${ }^{23,24}$ The body of evidence about false positive post treatment PET in pa-
Table 1. Demographic and clinical characteristics of PET-negative patients with Hodgkin's disease (N=73)

\begin{tabular}{ll}
\hline Characteristics & Values \\
\hline Median age (years) & 28 (range 12-80) \\
\hline Sex & 36 \\
Male & 37 \\
Female & 52 \\
\hline Histology & 10 \\
Nodular sclerosis & 1 \\
Mixed cellularity & 10 \\
Lymphocyte rich & \\
Not available & 3 \\
\hline Ann Arbor clinical stage & 46 \\
I & 16 \\
II & 8 \\
III & \\
IV & 38 \\
\hline B symptoms & 35 \\
\hline Yes & 24 \\
No & \\
\hline Treatment & \\
\hline Chemotherapy alone & \\
Chemotherapy and & \\
radiotherapy & \\
Follow-up after PET (months) & \\
Median & \\
\hline
\end{tabular}

tients with HD is constantly growing, ${ }^{12-14,18}$ and to gain accurate data FDG-avid lesions must be checked by biopsy, specially in previously unaffected region. $3,4,10,12-14,18,23,25$ Noninvasive alternative is to wait and repeat PET imaging in one or two months. ${ }^{24}$

This study has several limitations including a retrospective design, relatively small sample size reflecting the low incidence of $\mathrm{HD}$ and aggregation of patients with varying disease stages and treatment regimens. The PET equipment used was outdated lacking attenuation correction. Still, the high NPV indicates that coincidental PET scanning might be as reliable as dedicated PET or PET/CT systems, at least in patients with HD with a residual mass after the ini- 


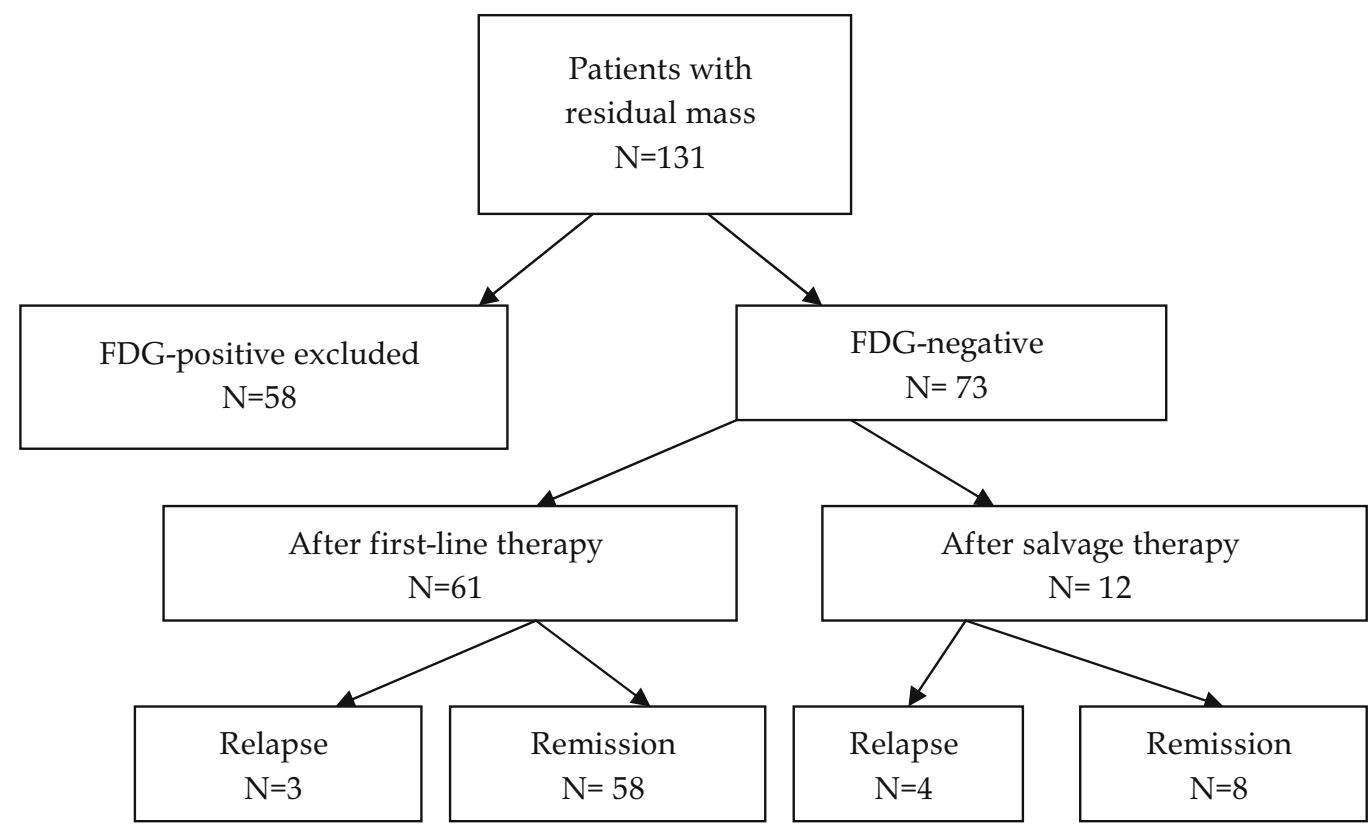

Figure 1. The flow diagram of primary or recurrent Hodgkin's disease (HD) patients with residual mass and FDGPET scan after first-line or salvage therapy.

tial therapy. This means that coincidence PET can be a viable alternative for those centres which do not have an easy access to dedicated PET or PET/CT systems, which is wildly used. ${ }^{8}$ In this constellation is a negative PET scan an important contribution in the management of patients and can provide reassurance to both, patients and their doctors, that disease is not active.

\section{References}

1. Israel O, Keidar Z, Bar-Shalom R. Positron emission tomography in the evaluation of lymphoma. Semin Nucl Med 2004; 3: 166-79.

2. Matasar MJ, Zelenetz AD. Overview of lymphoma diagnosis and management. Radiol Clin N Am 2008; 46: 175-98.

3. Hoskin PJ. FDG PET in the management of lymphoma: a clinical perspective. Eur J Nucl Med 2002; 29: 449-51.
4. Weihrauch MR, Re D, Scheidhauer K, Ansen S, Dietlein M, Bischoff S, et al. Thoracic positron emission tomography using $18 \mathrm{~F}$-fluorodeoxyglucose for the evaluation of residual mediastinal Hodgkin disease. Blood 2001; 98: 2930-4.

5. Naumann R, Vaic A, Beuthien-Baumann B, Bredow J, Kropp J, Kittner T, et al. Prognostic value of positron emission tomography in the evaluation of post-treatment residual mass in patients with Hodgkin's disease and non-Hodgkin's lymphoma. Br J Haematol 2001; 115: 793-800.

6. Lavely WC, Delbeke D, Greer JP, Morgan DS, Byrne DW, Price RR, et al. FDG PET in the followup management of patients with newly diagnosed Hodgkin and non-Hodgkin lymphoma after firstline chemotherapy. Int J Radiat Oncol Biol Phys 2003; 57: 307-15.

7. Spaepen K, Stroobants S, Dupont P, Thomas J, Vandenberghe P, Balzarini J, et al. Can positron emission tomography with [(18)F]fluorodeoxyglucose after first-line treatment distinguish Hodgkin's disease patients who need additional therapy from others in whom additional therapy would mean avoidable toxicity? $\mathrm{Br} J$ Haematol 2001; 115: 272-78. 
8. Nordin AJ, Secondino S, Rahim NA, Pedrazzoli $\mathrm{P}$, Siena S, Rossetti $\mathrm{C}$, et al. Imaging in nasopharyngeal carcinoma: the value of 18-Florine Fluorodeoxyglucose PET/CT in comparison to conventional imaging modalities CT and MRI. Radiol Oncol 2009; 43: 247-57.

9. Avazpour I, Roslan RE, Bayat P, Saripan MI, Nordin AJ, Raja Abdullah RSA. Segmenting CT images of bronchogenic carcinoma with bone metastases using PET intensity markers approach. Radiol Oncol 2009; 43: 180-6.

10. de Wit M, Bohuslavizki KH, Buchert R, Bumann D, Clausen M, Hossfeld DK. 18 FDG - PET following treatment as valid predictor for disease-free survival in Hodgkin's lymphoma. Ann Oncol 2001; 12: 29-37.

11. Zijlstra JM, Lindauer-van der Werf G, Hoekstra OS, et al. 18 F-fluoro-deoxyglucose positron emission tomography for post-treatment evaluation of malignant lymphoma: a systematic review. Haematologica 2006; 91: 522-9.

12. Terasawa T, Nihashi T, Hotta T, Nagai H. 18 F-FDG PET for posttherapy assessment of Hodgkin's disease and aggressive Non-Hodgkin's lymphoma: a systematic review. J Nucl Med 2008; 49: 13-21.

13. Schaefer NG, Taverna C, Strobel K, Wastl C, Kurrer M, Hany TF. Hodgkin disease: diagnostic value of FDG PET/CT after first line therapy - is biopsy of FDG - avid lesions still needed? Radiology 2007; 244: 257-62.

14. Poulou LS, Karianakis G, Ziakas PD. FDG PET scan strategies and long-term outcomes after firstline therapy in Hodgkin's disease. Eur J Radiol 2009; 70: 499-506.

15. Koga H, Sasaki M, Kuwabara Y, Nakagawa M, Hayashi K, Kaneko K, et al. Lesion detectability of a gamma camera based coincidence system with FDG in patients with malignant tumors: a comparison with dedicated positron emission tomography. Ann Nucl Med 2004; 18: 131-6.

16. Ak I, Blokland JA, Pauwels EK, Stokkel MP. The clinical value of 18F-FDG detection with a dualhead coincidence camera: a review. Eur J Nucl Med 2001; 28: 763-78.

17. Torizuka T, Nakamura F, Kanno T, Futatsubashi M, Yoshikawa E, Okada H, et al. Early therapy monitoring with FDG-PET in aggressive nonHodgkin lymphoma and Hodgkin's lymphoma. Eur J Nucl Med Mol Imaging 2004; 31: 22-8.

18. Schoder H, Moskowitz C. PET imaging for response assessment in lymphoma: potential and limitations. Radiol Clin N Am 2008; 46: 225-41.
19. Cheson BD, Horning SJ, Coiffier B, Shipp MA, Fisher RI, Connors JM, et al. Report of an international workshop to standardize response criteria for non-Hodgkin's lymphomas. J Clin Oncol 1999; 17: 1244-53.

20. Grošev D, Lončarić S, Vandenberghe S, Dodig D. Triple-head gamma camera PET: system overview and performance characteristics. Nucl Med Commun 2002; 23: 809-14.

21. Grošev D. Performance characteristics of gamma cameras - Reply. [Letter] Nucl Med Commun 2003; 24: 440 .

22. Kobe C, Dietlein M, Franklin J, Markova J, Lohri A, Amthauer $\mathrm{H}$, et al. Positron emission tomography has a high negative predictive value for progression or early relapse for patients with residual disease after first-line chemotherapy in advancedstage Hodgkin lymphoma. Blood 2008; 112: 398994.

23. Castellucci P, Zinzani P, Pourdehnad M, Alinari L, Nanni C, Farsad M, et al. ${ }^{18}$ F-FDG PET in malignant lymphoma: significance of positive findings. Eur J Nucl Med Mol Imaging 2005; 32: 749-56.

24. Kasamon YL, Jones RJ, Wahl RL. Integrating PET and PET/CT into the risk-adapted therapy of lymphoma. J Nucl Med 2007; 48 Suppl 1:19S-27S.

25. Barrington SF, O'Doherty MJ. Limitations of PET for imaging lymphoma. Eur J Nucl Med Mol Imaging 2003; 30: S117-27. 